\title{
CHARM HADRONIC DECAYS FROM FOCUS: LESSONS LEARNT
}

\author{
STEFANO BIANCO* \\ Laboratori Nazionali di Frascati dell'INFN, v. E. Fermi 40 \\ Frascati, 00044 Italy \\ stefano.bianco@lnf.infn.it \\ DANIELE PEDRINI \\ INFN Milano Bicocca, Edificio U2 - Piazza della Scienza 3 \\ I-20126 Milano - Italy \\ daniele.pedrini@mib.infn.it \\ ALBERTO REIS \\ Centro Brasileiro de Pesquisas Físicas, Rua Dr. Xavier Sigaud, 150 - Urca \\ Rio de Janeiro - RJ - Brasil 22290-180 \\ alberto@cbpf.br
}

\begin{abstract}
The FOCUS photoproduction experiment took data in the ninenties and produced a wealth of results in charm physics. Some of the studies were seminal for contemporary experiments, and even paved the way for the technology of many charm and beauty analysis tools.
\end{abstract}

Keywords: charm; hadronic decays; Photoproduction.

PACS numbers: 14.65.Dw; 14.40.Lb

\section{Introduction}

A retrospective discussion of selected FOCUS hadronic decays results which have interest and implications for ongoing and future studies in charm and beauty decays is presented. Topics which had long lasting effects on heavy flavour physics are discussed, such as the measurement of $y_{C P}$ parameter in $\mathrm{D}$ mixing, the study of T-odd correlations, and the four-body amplitude analysis.

Data were collected 1996-1997 with the FOCUS spectrometer at the Fermilab Tevatron Wide Band photon beam. The FOCUS spectrometer ${ }^{1}$ was successor to E687 and designed to study charm particles produced by $200 \mathrm{GeV}$ photons using a fixed target spectrometer with vertexing, cerenkov detectors, em calorimeters, and muon id capabilities. Member groups of the FOCUS collaboration were from USA, Italy, Brazil, Mexico, Korea.

*Presenter 


\section{The $y_{C P}$ parameter}

The study ${ }^{2}$ consisted of a comparison of the lifetime of a CP even final state, $D^{0} \rightarrow K^{-} K^{+}$, to the lifetime of a CP mixed decay, $D^{0} \rightarrow K^{-} \pi^{+}$. The lifetime measurements (Fig. 1) were made using high signal-to-background $D^{0}$ samples consisting of 10331 decays into $K^{-} K^{+}$, and 119738 decays into $K^{-} \pi^{+}$. The measurement of lifetime differences corresponded to a direct measurement of the mixing parameter $y_{\mathrm{CP}}=(\Gamma(\mathrm{CP}$ even $)-\Gamma(\mathrm{CP}$ odd $)) /(\Gamma(\mathrm{CP}$ even $)+\Gamma(\mathrm{CP}$ odd $))=$ $0.0342 \pm 0.0139 \pm 0.0074$.

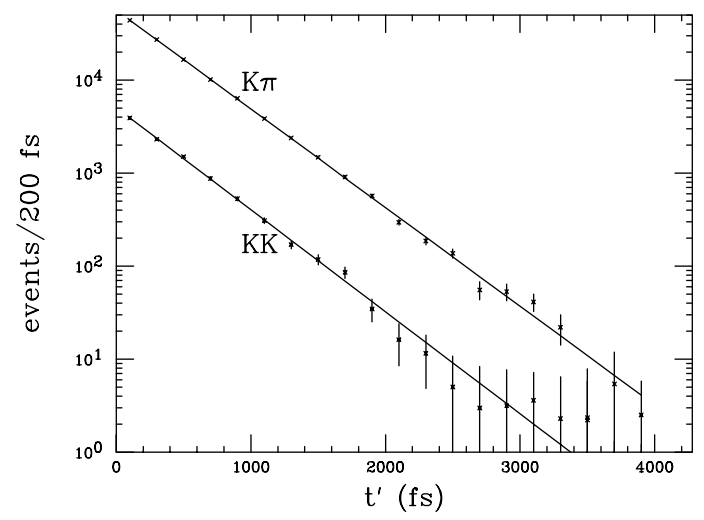

Fig. 1. Signal versus reduced proper time for $D^{0} \rightarrow K^{-} \pi^{+}$and $K^{-} K^{+}$requiring $W_{\pi}-W_{K}>4$ and $\ell / \sigma>5$. The fit is over 20 bins of $200 \mathrm{fs}$ bin width. The data is background subtracted and includes the (very small) Monte Carlo correction.

Such a first evidence for nonzero mixing was followed by higher-statistics measurements from B-factories. Fig.2 from a 2003 review paper ${ }^{3}$ shows the FOCUS measurement compared to the existing studies. With a moderate significance due to low statistics, FOCUS showed evidence for a nonzero lifetime difference. Recent results from BABAR and BELLE have solidly established the observation of D mixing. Algorithms and selections used by FOCUS may be used by forward-geometry experiment $\mathrm{LHCb}$ at the CERN LHC with much larger statistics very soon.

\section{T-odd correlations}

Triple-product correlations of the form $\overrightarrow{v_{1}} \cdot\left(\overrightarrow{v_{2}} \times \overrightarrow{v_{3}}\right)$, where each $\overrightarrow{v_{i}}$ is a spin or momentum, are odd under time reversal $(T)$. By the $C P T$ theorem, a nonzero value for these correlations would also be a signal of $C P$ violation. A nonzero tripleproduct correlation is evidenced by a nonzero value of the asymmetry

$$
A_{T} \equiv \frac{\Gamma\left(\overrightarrow{v_{1}} \cdot\left(\overrightarrow{v_{2}} \times \overrightarrow{v_{3}}\right)>0\right)-\Gamma\left(\overrightarrow{v_{1}} \cdot\left(\overrightarrow{v_{2}} \times \overrightarrow{v_{3}}\right)<0\right)}{\left.\left.\left.\Gamma \overrightarrow{v_{2}} \times \overrightarrow{v_{3}}\right)>0\right)+\Gamma\left(\overrightarrow{v_{1}}\right)<0\right)}
$$




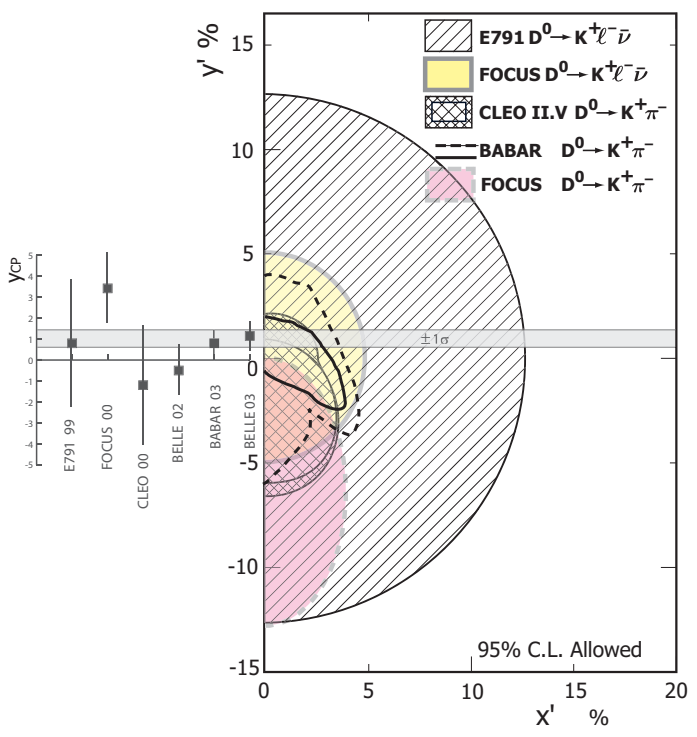

Fig. 2. Summary of $x^{\prime}, y^{\prime}, y_{C P}$ measurements in 2003.

where $\Gamma$ is the decay rate for the process. There is a well-known technical complication: strong phases can produce a nonzero value of $A_{T}$, even if the weak phases are zero, that is $C P$ and $T$ violation are not necessarily present. Thus, strictly speaking, the asymmetry $A_{T}$ is not in fact a $T$-violating effect. Nevertheless, one can still obtain a true $T$-violating signal which takes care of the complications due to strong phases by measuring a nonzero value of

$$
A_{\text {Tviol }} \equiv \frac{1}{2}\left(A_{T}-\overline{A_{T}}\right)
$$

where $\overline{A_{T}}$ is the $T$-odd asymmetry measured in the $C P$-conjugate decay process. This study was inspired by a paper of Ikaros Bigi ${ }^{5}$. In his paper Bigi suggested a search for $T$ violation by looking at the triple-product correlation (using the momenta of the final state particles) in the decay mode $D^{0} \rightarrow K^{-} K^{+} \pi^{-} \pi^{+}$. Such a correlation must necessarily involve at least four final-state particles. This can be understood by considering the rest frame of the decaying particle and invoking momentum conservation. The number of independent three-momenta is one less than the number of final-state particles, so a triple product composed entirely of momenta requires four particles in the final state. We calculate $A_{\text {Tviol }}$ for the decay modes $D^{0} \rightarrow K^{-} K^{+} \pi^{-} \pi^{+}$and $D_{(s)}^{+} \rightarrow K_{S}^{0} K^{+} \pi^{-} \pi^{+}$using data from the FOCUS experiment, finding, with about $800 D^{0}$ events $^{4}, A_{\text {Tviol }}\left(D^{0}\right)=0.010 \pm 0.057$ (stat. $) \pm$ 0.037 (syst.), and, with about $500 D^{+}$and $500 D_{s}^{+}$events, $A_{\text {Tviol }}\left(D^{+}\right)=0.023 \pm$ 0.062 (stat.) \pm 0.022 (syst.), and $A_{\text {Tviol }}\left(D_{s}^{+}\right)=-0.036 \pm 0.067$ (stat.) \pm 0.023 (syst.).

In 2010 BABAR published new limits ${ }^{6}$ by using the same FOCUS formalism. With $4.710^{4}$ events, BABAR measured $A_{\text {Tviol }}\left(D^{+}\right)=0.0010 \pm 0.0051$ (stat.) \pm 
0.0044(syst.). Another order of magnitude limit reduction is expected with the Frascati and KEK super-b factories data samples. For an updated discussion see ${ }^{7}$.

\section{Four-body amplitude analysis}

FOCUS published the first study ${ }^{8},{ }^{9},{ }^{10}$ with a complete 4-body formalism of KK $\pi \pi$, $\mathrm{KKK} \pi, \pi \pi \pi \pi$. In this conference report only the $\pi \pi \pi \pi$ channel is discussed, with $6.3 \mathrm{k}$ events. This decay has a direct connection to decays used for extraction of CKM parameters in B decays such as the angle $\alpha$ from $B \rightarrow \rho^{0} \rho^{0}$.

A simple isobar model was used with a total of nine amplitudes and 16 free parameters: $D \rightarrow \rho^{0} \rho^{0} ; D \rightarrow a_{1} \pi, a 1 \rightarrow \rho^{0} \pi ; D \rightarrow R \pi \pi, R \equiv$ $\left(\sigma, \rho^{0}, f_{0}(980), F_{2}(1270)\right)$. As customary in all isobar models, final state interactions (FSI) are not fully accounted for. Several helicity states were considered in the fit functions. The study showed how the dominant contribution to the $\pi \pi \pi \pi$ state comes from the $a_{1}^{+}(1260) \pi^{-}$mode, which accounts for over $60 \%$ of the total decay rate. The remaining part of the decay rate is equally divided between $\rho^{0} \rho^{0}$ and the quasi-three-body modes $D \rightarrow R \pi \pi$. The scalar component is dominated by the decay $D^{0} \rightarrow \sigma \pi \pi$. The fit get significantly worse if the $\sigma \pi \pi$ amplitude is replaced by an uniform non-resonant component.

Most important, although the one-dimensional projections suggest a good fit, the goodness-of-fit tested on the full 5-dimensional phase space is poor. This indicates that other effects need to be taken into account for a more realistic model. One important ingredient is the final state interaction (FSI), which could introduce energy-dependent phases. One needs to build a model for the FSI based on chiral perturbation theory, but this is rather challenging even for three-body final states. Another effect that could play a role is the Bose-Einstein correlation between the two pairs of identical pions.

The final message is that, at least in four-body decays, the strong interaction phases are still not under control, which makes the extraction of weak phases governing CP violation rather uncertain.

\section{Epilogue: on the importance of Dalitz analysis in the extraction of CKM parameters}

Dalitz plot analysis is fundamental for a meaningful investigation of decay modes in which resonances are present. As an example, FOCUS showed ${ }^{11}$ in the analysis of the decay mode $D^{0} \rightarrow K_{s} K^{+} K^{-}$that a contamination from $f_{0}(980)$ under the $\phi(1020)$ signal is always present if a simple mass cut is applied. This, however, changes the assignment of the final state, since $K_{s} \phi$ is a CP-odd state, while $K_{s} f_{0}$ is CP-even.

These considerations forced, in early 2000s, the B-factory experiments to revisit their analyses of some decay modes such as $B^{0} \rightarrow K_{s} K^{-} K^{+}$. Nowadays these analyses are performed with the Dalitz plot technique. 


\section{Conclusions}

FOCUS/E687 was a pioneering expedition towards the charm unexplored territories, the first experiment which had important measurements for all weakly decaying charm hadrons including weak lifetimes up to the $\Omega_{c}$, thus showing us that some nonperturbative QCD effects could be understood. FOCUS gave hints of unexpected phenomena such as a positive value for the $y_{C P}$ parameter in charm mixing, which is still today debated subject of studies, and possibly the only mixing parameter significantly different from zero observed so far. FOCUS did pioneering work for three-body final states to help us in the future to find CPV in D decays, and to help understand CP searches in B decays.Future searches for CPV from New Physics need much more experimental and theoretical work. Finally, FOCUS did pioneering work in $D \rightarrow 4 h$, useful for the future and showing that more theoretical work is needed. From the experimental side, while BaBar/BELLE reached the $1 \%$ level in $\mathrm{CPV}$ limits, much more statistics is needed to reach the $0.1 \%$ level where NP can generate effects.

Three FOCUS hadronic decays results have been singled out and discussed. The results have implications on upcoming new results from the contemporary charm and beauty experiments. Algorithm and selection used by FOCUS in the study of $y_{C P}$ parameter in $\mathrm{D}$ mixing may be used by forward-geometry experiment $\mathrm{LHCb}$ with much larger statistics. The formalism for the search of $\mathrm{CP}$ violation via T-odd correlations was developed, and first limit on KK final state published, which was followed in 2010 by the new limit from BABAR in 2010 using the same formalism. The first study with a complete 4-body formalism of $\mathrm{KK} \pi \pi, \mathrm{KKK} \pi, \pi \pi \pi \pi$ provided a direct connection to decays used for extraction of CKM angle $\alpha$ in B decays: the poor 5D fit quality which emerges in high-statistics studies implies the need for a better understanding of nonresonant component. Finally, Dalitz plot analyses from FOCUS showed the importance of studying the resonant structure of hadronic decays: S-waves cannot be taken out with a simple mass cut.

\section{Acknowledgments}

The authors acknowledge valuable comments and discussions with I.I. Bigi. This research was supported in part by the Italian Istituto Nazionale di Fisica Nucleare and Ministero della Istruzione Università e Ricerca, .

\section{References}

1. P. L. Frabetti et al. [E-687 Collaboration], Nucl. Instrum. Meth. A 320, 519 (1992).

2. J. M. Link et al. [FOCUS Collaboration], Phys. Lett. B 485, 62 (2000) [arXiv:hepex/0004034].

3. S. Bianco, F. L. Fabbri, D. Benson and I. Bigi, Riv. Nuovo Cim. 26N7, 1 (2003) [arXiv:hep-ex/0309021].

4. J. M. Link et al. [FOCUS Collaboration], Phys. Lett. B 622, 239 (2005) [arXiv:hepex/0506012]. 
5. I.I. Bigi, in Proceedings of KAON2001: International Conference on $C P$ Violation, Pisa, Italy, 12-17 Jun 2001, p.417 (hep-ph/0107102).

6. P. del Amo Sanchez et al. [The BABAR Collaboration], Phys. Rev. D 81, 111103 (2010) [arXiv:1003.3397 [hep-ex]].

7. D.Pedrini, in The Heavy Flavor Averaging Group (D. Asner et al.), arXiv:1010.1589v1.

8. J. M. Link et al. [FOCUS Collaboration], Phys. Rev. D 75, 052003 (2007) [arXiv:hepex/0701001].

9. J. M. Link et al. [FOCUS Collaboration], Phys. Lett. B 610, 225 (2005) [arXiv:hepex/0411031].

10. J. M. Link et al. [FOCUS Collaboration], Phys. Lett. B 575, 190 (2003) [arXiv:hepex/0308054].

11. This argument was first pointed out by D. Pedrini, D. Pedrini, Presented at 1st International Workshop on Frontier Science: Charm, Beauty, and CP, Frascati, Rome, Italy, 6-11 Oct 2002. 